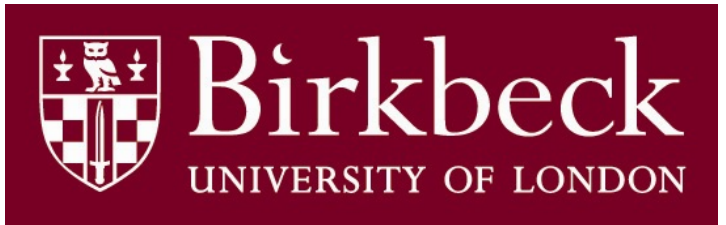

School of Economics, Mathematics and Statistics

BWPEF 0604

\title{
Fractional Diffusion Models of Option Prices in Markets with Jumps
}

\author{
Álvaro Cartea \\ Birkbeck, University of London \\ Diego del-Castillo-Negrete \\ Oak Ridge National Laboratory, U.S.
}

August 2006 


\title{
Fractional Diffusion Models of Option Prices in Markets with Jumps Forthcoming: Physica A
}

\author{
Álvaro Cartea \\ Birkbeck College, University of London, UK \\ Email: a.cartea@bbk.ac.uk \\ Diego del-Castillo-Negrete \\ Oak Ridge National Laboratory, US \\ Email: delcastillod@ornl.gov
}

August 11, 2006

\begin{abstract}
Most of the recent literature dealing with the modeling of financial assets assumes that the underlying dynamics of equity prices follow a jump process or a Lévy process. This is done to incorporate rare or extreme events not captured by Gaussian models. Of those financial models proposed, the most interesting include the CGMY, KoBoL and FMLS. All of these capture some of the most important characteristics of the dynamics of stock prices. In this article we show that for these particular Lévy processes, the prices of financial derivatives, such as European-style options, satisfy a fractional partial differential equation (FPDE). As an application, we use numerical techniques to price exotic options, in particular barrier options, by solving the corresponding FPDEs derived.
\end{abstract}


Keywords: Fractional-Black-Scholes, Lévy-Stable processes, FMLS, KoBoL, CGMY, fractional calculus, Riemann-Liouville fractional derivative, barrier options, down-and-out, up-and-out, double knock-out.

\section{Introduction}

A problem of significant interest in finance is the pricing of financial instruments that derive their value from financially traded assets such as stocks. Among the first systematic treatments of this problem was the pioneering work of Black, Scholes and Merton who proposed the widely known, and extensively used, Black-Scholes (BS) model, Ref. [23]. The BS model rests on the assumption that the natural logarithm of the stock price $S_{t}$ follows a random walk or diffusion with deterministic drift:

$$
d\left(\ln S_{t}\right)=\left(\mu-\frac{1}{2} \sigma^{2}\right) d t+\sigma d B_{t}
$$

where $\mu>0$ is the average compounded growth of the stock $S_{t} ; d B_{t}$ is the increment of Brownian motion which is assumed to have the Normal or Gaussian distribution; i.e. $d B_{t} \sim N(0, d t)$; and $\sigma \geq 0$ is the volatility of the returns from holding $S_{t}$.

Once a stochastic process for the evolution of prices has been specified, it is possible to address the question of how options on traded stocks such as $S_{t}$ are priced. The simplest examples of options are known as European calls and puts. A European call gives the owner of the option the right, but not the obligation, to buy a unit of stock $S_{t}$ at a known future time $T$ for a pre-specified price $K$, known as the strike price. Similarly, a European put gives the owner of the option the right, but not the obligation, to sell a unit of stock $S_{t}$ at a future known date $T$, for a pre-specified

price $K$. More generally, European-style options refer to options that may only be exercised at a future known date $T$. Moreover, an American option is like holding a European option but with the extra flexibility that it can be exercised at any time until the expiry date.

According to the BS model, the price of a European-style option $V(S, t)$, written 
on the traded asset $S_{t}$, satisfies the partial differential equation (PDE)

$$
\frac{\partial V(S, t)}{\partial t}+\frac{1}{2} \sigma^{2} S^{2} \frac{\partial^{2} V(S, t)}{\partial S^{2}}+r S \frac{\partial V(S, t)}{\partial S}=r V(S, t)
$$

where $r$ is the risk-free rate, Ref. [34]. The BS equation may also be written as an advection-diffusion type equation by making the change of variable $x_{t}=\ln S_{t}$ in Eq. (2)

$$
\frac{\partial V(x, t)}{\partial t}+\frac{1}{2} \sigma^{2} \frac{\partial^{2} V(x, t)}{\partial x^{2}}+\left(r-\frac{1}{2} \sigma^{2}\right) \frac{\partial V(x, t)}{\partial x}=r V(x, t)
$$

One of the most significant shortcomings of the BS model is that financial data shows that Gaussian shocks underestimate the probability that stock prices exhibit large movements or jumps over small time steps. To address this issue, a number of extensions or alternative stochastic shocks to the random walk in Eq. (1) have been proposed. In broad terms, these models have either assumed a two-factor model where the dynamics of the stock price follow Eq. (1) and the volatility $\sigma$ follows a stochastic process, or a stock price which follows a jump process or a Lévy process (i.e. a jump process with independent and stationary increments).

It is well-known in the literature that when the Brownian motion component in Eq. (1) is substituted by a Lévy process, the pricing Eq. (3) becomes a partialintegro-differential equation (PIDE), Ref. [32]. PIDEs are introduced to capture the non-locality induced by the jumps in the Lévy process. Our contribution in this article is twofold. First, we show that for European-style options written on assets, that follow some of the most advocated jump models in the financial literature, one can write the general PIDE as a fractional partial differential equation (FPDE), which are a subset of the class of pseudo-differential equations. Second, we solve numerically this FPDE to price exotic financial instruments such as barrier options.

Previous work on numerical methods for PIDEs include Ref. [11] where a finitedifference scheme was proposed for option pricing in jump diffusion and exponential Lévy models. Other methods include the use of fast Fourier transform to price pathindependent European-style options Ref. [7]. As an alternative to these methods, here we propose techniques from fractional calculus. In our approach the problem of the truncation (localization) of the non-local operators to finite size domains is 
circumvented with the use of regularized fractional derivative operators. For the discretization of the PIDE we use the Grünwald-Letnikov representation of the fractional derivative that provides an efficient, relatively easy to implement finite-difference numerical scheme. The truncation and finite-difference scheme used here are based on the numerical method originally proposed in Refs. $[13,12]$ for the solution of fractional diffusion equations. The pricing of barrier options for exponentially damped processes has been studied analytically and numerically in Refs. $[2,11]$. As an application of our fractional calculus approach, here we consider the less studied problem of barrier options for finite moment log-stable (FMLS) processes.

Applications of fractional calculus in finance include Cartea Ref. [9]. In this article the author shows that classical hedging strategies, ie risk minimizing, based on 'localised' information of the value of a hedge portfolio, for instance those based on the information provided by the delta and gamma of the portfolio, can be substantially improved by employing fractional or 'non-local' operators. ${ }^{1}$ The author extends the classical idea that a market player who sold an option written on the underlying $S_{t}$, can hedge it by setting up a portfolio consisting of the short option plus an amount of $S_{t}$ plus another option also written on $S_{t}$. Rather than making the portfolio deltaneutral and gamma-neutral over a time step $\Delta t$, ie choose the amounts of $S_{t}$ and second option in the portfolio so that the first and second derivative of the value of the portfolio are zero (see Ref. [1]), the fractional strategy makes the portfolio deltaneutral and neutral to a fractional derivative (that includes the gamma derivative as a particular case) over $\Delta t$. The intuition behind why the fractional strategy outperforms the classical approach of delta-gamma-hedging relies on the non-local nature of the fractional operator. It must be pointed out that over a time step $\Delta t$ the stock price $S_{t}$ can diffuse and/or jump to values $S_{t+\Delta t}$ 'far away' from $S_{t}$ making the use of localized information of the portfolio at $S_{t}$ less relevant. The fractional derivative 'weighs' information of the portfolio over a range of values of the underlying rather than looking at localized information. Finally, for other applications of fractional calculus in finance see Refs. [31, 18].

\footnotetext{
${ }^{1}$ The delta of a financial instrument is the first derivative of the value of the instrument with respect to the underlying. For example, the delta of a European-style option $V(S, t)$ is given by $\partial V(S, t) / \partial S$. Similarly, the gamma is the second derivative, $\partial^{2} V(S, t) / \partial S^{2}$.
} 
The rest of the article is structured as follows: Section 2 reviews basic concepts of Lévy processes and their use in financial modeling. Section 3 presents concepts of fractional calculus and derives the FPDEs satisfied by the value of European options written on assets that follow the particular processes presented in section 2. Section 4 prices barrier options by numerically solving the FPDEs derived in section 3. Finally, section 5 concludes and discusses further applications of this work, such as the pricing of American and other exotic options.

\section{Lévy processes and stock price models}

Examples where it has been assumed that share prices follow jump processes include: the early work of Press Ref. [28]; Merton's Jump Diffusion model Ref. [23]; and the work of Mantegna and Stanley, see Refs. [20, 21, 22], which builds on the work of Mandelbrot Ref. [19] to show that Truncated Lévy Flights can be very successful at capturing the high-frequency empirical probability distribution of the S\&P 500 index. Based on this work, Koponen Ref. [15] and Boyarchenko and Levendorskii Ref. [3] proposed the use of modified LS (also known as Lévy- $\alpha$-stable) processes to model the dynamics of securities. This modification introduced a damping effect in the tails of the LS distribution to ensure finite moments and gain mathematical tractability; these models are known in the mathematical finance literature as KoBoL processes. Finally, motivated by the most important properties of the dynamics of share prices including size and frequency of both positive and negative jumps in the stock price movements, Carr, Geman, Madan and Yor proposed the CGMY process Ref. [6]. This is essentially the same model as the KoBoL, and has quickly become one of the most widely used models for equity prices.

Although the class of Lévy processes is vast, one can characterize them in a very compact way via the characteristic function of the process. More precisely, a timedependent random variable $X_{t}$ is a Lévy process if and only if it has independent and stationary increments with log-characteristic function given by the Lévy-Khintchine 
representation

$$
\ln \mathbb{E}\left[e^{i \xi X_{t}}\right] \equiv t \Psi(\xi)=m i t \xi-\frac{1}{2} \sigma^{2} t \xi^{2}+t \int_{\mathbb{R} \backslash\{0\}}\left(e^{i \xi x}-1-i \xi h(x)\right) W(d x),
$$

where $m \in \mathbb{R}, \sigma \geq 0, h(x)$ is a truncation function, the Lévy measure $W$ must satisfy

$$
\int_{\mathbb{R}} \min \left\{1, x^{2}\right\} W(d x)<\infty
$$

and $\Psi(\xi)$ is known as the characteristic exponent of the Lévy process, Ref. [14]. A Lévy process can be seen as a combination of a drift component, and two independent processes: a Brownian motion component and a jump component. These three components are completely determined by the Lévy-Khintchine triplet $\left(m, \sigma^{2}, W\right)$. The Lévy measure $W$ is responsible for the behavior of the jump component of $X_{t}$ and determines the frequency and magnitude of jumps. If the Lévy measure is of the form $W(d x)=w(x) d x, w(x)$ is called the Lévy density.

To understand how Lévy processes are incorporated in the derivatives pricing models, it is instructive to recall how Gaussian shocks are built into the standard BS framework. To find the fair, or arbitrage free, price of financial instruments that derive their value from an underlying stock price $S_{t}$, it is necessary to express the dynamics of $S_{t}$ under what is known as the risk-neutral measure or equivalent martingale measure (EMM), Ref. [32]. For example, in the BS model, this means that the random walk followed by $S_{t}$ under the EMM is

$$
d\left(\ln S_{t}\right)=\left(r-\frac{1}{2} \sigma^{2}\right) d t+\sigma d B_{t}^{Q}
$$

where $\sigma>0, r$ is the risk-free rate and $d B_{t}^{Q}$ is the increment of a Brownian motion under the EMM. Note that this random walk is similar to Eq. (1) but the drift now contains the risk-free rate $r$ and while the stochastic component is again Brownian motion, we stress that it is under the risk-free measure by using the superscript $Q$.

In the BS model, the price of a European call option $C(S, t ; K, T)$ struck at $K$, expiring at $T$ and with payoff $\max \left(S_{T}-K, 0\right)$, can be calculated by either taking expectations of the payoff discounted by the risk-free rate

$$
C(S, t ; K, T)=e^{-r(T-t)} \mathbb{E}^{Q}\left[\max \left(S_{T}-K, 0\right)\right]
$$


when $S_{t}$ follows Eq. (6), or by solving the BS PDE Eq. (2) subject to the appropriate boundary conditions, Ref. [34]. The BS model is one of the few where there are closed-form solutions for the value of European-style options. However, as we shall see below, once a more realistic random walk for the risk-neutral dynamics of the stock price $S_{t}$ is assumed, the pricing of instruments, more complex than European call and put options, is not straightforward. In such cases, one must resort to numerical methods to solve the analogue PDE to the BS Eq. (2) to price other type of financial instruments.

Based on the poor empirical performance of the BS model, much of the recent financial literature proposes to replace the Brownian shocks in Eq. (1) with a Lévy process so that

$$
d\left(\ln S_{t}\right)=\mu d t+d L_{t}^{P},
$$

where we denote the increments of the Lévy process, under the physical or historical measure $P$, by $d L_{t}^{P}$. As in the Brownian motion case, pricing of instruments is performed under a chosen EMM that will no longer be unique due to the presence of jumps introduced by the Lévy process $L_{t}$. The literature proposes a number of ways of choosing an EMM under which pricing of instruments is performed. One of the most popular approaches has been to assume that under the EMM the stock price stays within the family of Lévy processes (not necessarily the same one driving the historical process) and this is the approach adopted here. For a rigorous treatment of the connection between historical and risk-neutral measures see Refs. [5, 10].

Therefore, for the purposes of pricing financial instruments, it is further assumed that, under the risk-neutral measure, the stock price follows a geometric Lévy process

$$
d\left(\ln S_{t}\right)=(r-v) d t+d L_{t}
$$

with solution

$$
S_{T}=S_{t} e^{(r-v)(T-t)+\int_{t}^{T} d L_{u}},
$$

where $r$ is the risk-free rate, $v$ is a convexity adjustment so that $\mathbb{E}^{Q}\left[S_{T}\right]=e^{r(T-t)} S_{t}$, and $d L_{t}$ is the increment of a Lévy process under the EMM Ref. [10]. Note that Eq. (6) is a particular case of Eq. (8) when the Lévy process $L_{t}$ has triplet $\left(0, \sigma^{2}, 0\right)$ 
and $v=\frac{1}{2} \sigma^{2}$. Below we discuss in detail the particular choices of Lévy processes we are interested in: LS, CGMY and KoBoL.

\section{$2.1 \quad$ LS processes}

For an LS process, the Lévy density is given by

$$
w_{L S}(x)= \begin{cases}D q|x|^{-1-\alpha} & \text { for } x<0 \\ D p x^{-1-\alpha} & \text { for } x>0\end{cases}
$$

where $D>0, p, q \in[-1,1]$ with the restriction $p+q=1$ and $0<\alpha \leq 2$. Using Eq. (4) yields the characteristic exponent of an LS process in terms of the parameters, $\alpha, \sigma, \beta$ and $m$ :

$$
\Psi_{L S}(\xi)=-\frac{1}{2} \sigma^{\alpha}|\xi|^{\alpha}\{1-i \beta \operatorname{sign}(\xi) \tan (\alpha \pi / 2)\}+i m \xi \quad \text { for } \alpha \neq 1 .
$$

An equivalent, more convenient, expression of $\Psi_{L S}(\xi)$, which we use below, is

$$
\Psi_{L S}(\xi)=-\frac{\sigma^{\alpha}}{4 \cos (\alpha \pi / 2)}\left\{(1-\beta)(i \xi)^{\alpha}+(1+\beta)(-i \xi)^{\alpha}\right\}+i m \xi
$$

If the random variable $X$ belongs to an LS distribution with parameters $\alpha, \sigma, \beta$ and $m$ we write $X \sim \operatorname{LS}_{\alpha}(\sigma, \beta, m)$. The parameter $\alpha$ is known as the stability index; $\sigma$ is a scaling parameter; $-1 \leq \beta \leq 1$ is a skewness parameter with $\beta=p-q$ and $m$ is a location parameter. Moreover, we point out that when $\beta=-1$ (resp. $\beta=1$ ) the random variable $X$ is maximally skewed to the left (resp. right). For $\alpha=2$ and $\beta=0$ the Gaussian case is obtained and apart from the case $\alpha=2$, the random variable $X$ possesses infinite variance. Although there are strong theoretical grounds that support the use of LS processes in financial modeling, the infinite moments property makes it difficult to implement it from a mathematical and financial viewpoint Ref. [22]. However, for maximally skewed LS processes with $\beta=-1$, the Laplace transform of the process $X_{t} \sim \operatorname{LS}_{\alpha}\left(t^{1 / \alpha} \sigma,-1, a\right)$ exists. This choice of parameters gives rise to an interesting financial model known as the finite moment log-stable (FMLS), Ref. [8]. A particular feature of the FMLS process is that it only exhibits downwards jumps, whilst upwards movements have continuous paths. This is straightforward to see by inspecting the Lévy density in Eq. (10) evaluated at $q=1$ and $p=0$, i.e. $\beta=-1$. 


\subsection{CGMY and KoBoL processes}

We recall that to circumvent the infinite variance limitation of LS processes and to ensure the existence of moments of all orders, it was proposed to truncate the tails of the LS distribution Refs. [20, 22]. This approach was extended by introducing an exponential damping in the Lévy density Eq. (10) to yield a more tractable formulation of the characteristic function of the stochastic process. Two 'damped' LS processes have been proposed in the financial literature: CGMY and KoBoL.

The CGMY process is a pure jump Lévy process (i.e. it has no Brownian motion component) with Lévy measure $W(d x)=w_{C G M Y}(x) d x$

$$
w_{C G M Y}(x)= \begin{cases}C \frac{e^{-G|x|}}{|x|^{1+Y}} & \text { for } x<0 \\ C \frac{e^{-M x}}{x^{1+Y}} & \text { for } x>0\end{cases}
$$

Substituting it in the Lévy-Khintchine representation Eq. (4) with $m=0$, and evaluating the integral, we obtain the characteristic exponent

$$
\Psi_{C G M Y}(\xi)=C \Gamma(Y)\left\{(M-i \xi)^{Y}-M^{Y}+(G+i \xi)^{Y}-G^{Y}\right\}
$$

Here $C>0, G \geq 0, M \geq 0$ and $Y<2$. The parameter $C$ may be viewed as a measure of the overall level of activity. The parameters $G$ and $M$ control the exponential decay of the left and right tail respectively and the distribution is symmetric when $G=M$.

The KoBoL process is also a pure jump Lévy process, very similar to the CGMY, with Lévy density

$$
w_{\text {KOBOL }}(x)= \begin{cases}D q|x|^{-1-\alpha} e^{-\lambda|x|} & \text { for } x<0, \\ D p x^{-1-\alpha} e^{-\lambda x} & \text { for } x>0\end{cases}
$$

therefore the characteristic exponent becomes

$$
\begin{aligned}
& \Psi_{\text {KOBOL }}(\xi)=\frac{1}{2} \sigma^{\alpha}\left\{p(\lambda-i \xi)^{\alpha}+q(\lambda+i \xi)^{\alpha}-\lambda^{\alpha}\right\} \\
& \Psi_{\text {KOBOL }}(\xi)=\frac{1}{2} \sigma^{\alpha}\left\{p(\lambda-i \xi)^{\alpha}+q(\lambda+i \xi)^{\alpha}-\lambda^{\alpha}-i \xi \alpha \lambda^{\alpha-1}(q-p)\right\}
\end{aligned}
$$

for $0<\alpha<1$ and for $1<\alpha \leq 2$ respectively. The parameter $\lambda>0$ plays the same role as $G$ and $M$ do in the CGMY model, while the other parameters perform a similar function to those in the LS process. The main differences between the CGMY and 
KoBoL are that in the CGMY the parameter $Y<2$ while for the KoBoL $0<\alpha \leq 2$. Moreover, the skewness in the CGMY is controlled by $G$ and $M$ while in the KoBoL by $p$ and $q$.

\section{A fractional calculus approach to option pricing}

In this section we derive the FPDEs satisfied by options written on assets that follow the Lévy processes presented above. To establish the connection between the fractional pricing equations and these processes, we first note that if the log-stock process follows Eq. (8), where the characteristic exponent of the Lévy process $L_{t}$ is $\Psi(\xi)$, then the Fourier transform, denoted by

$$
\hat{f}(\xi)=\int_{-\infty}^{\infty} e^{i \xi x} f(x) d x=\mathcal{F}\{f(x)\},
$$

of the value of a European-style option $\hat{V}(\xi, T)$, written on $S_{t}$, satisfies

$$
\frac{\partial \hat{V}(\xi, t)}{\partial t}=[r+i \xi(r-v)-\Psi(-\xi)] \hat{V}(\xi, t),
$$

with boundary condition $\hat{V}(\xi, T)=\hat{\Pi}(\xi, T)$.

One can prove this result by looking at the infinitesimal generator of the Lévy process Ref. [10]. Here we provide an alternative and straightforward proof in Appendix A where we use the fact that the characteristic function of $\ln S_{T}$, using Eq. (9), is given by

$$
\mathbb{E}^{Q}\left[e^{i \xi \ln S_{T}}\right]=e^{i \xi \ln S_{t}+i \xi(T-t)(r-v)+(T-t) \Psi(\xi)}
$$

We stress that Eq. (17) is general in the sense that it encompasses all Lévy process, with finite exponential moments, and not only the ones described in Section 2. We will use Eq. (17) to derive the FPDEs satisfied by European-style options written on assets that follow the random walk Eq. (8), via its expression in Fourier space Eq. (18), with different choices of $d L_{t}$ and its corresponding convexity adjustment $v$. In particular, we assume that the underlying risk-neutral dynamics of an asset $S_{t}$ follow either an FMLS (or maximally skewed LS process), CGMY or KoBoL process. 
In Appendix B we review concepts of fractional calculus whilst here we present the basic definition and notation of fractional derivatives. We define the RiemannLiouville (RL) fractional derivative of the function $f$ as follows, Ref. [27]. The left and right fractional derivatives, of order $\gamma$ of the function $f$, are respectively given by

$$
{ }_{a} D_{x}^{\gamma} f(x)=\frac{1}{\Gamma(n-\gamma)} \frac{\partial^{n}}{\partial x^{n}} \int_{a}^{x}(x-y)^{n-\gamma-1} f(y) d y \quad n-1 \leq \gamma<n,
$$

and

$$
{ }_{x} D_{b}^{\gamma} f(x)=\frac{(-1)^{n}}{\Gamma(n-\gamma)} \frac{\partial^{n}}{\partial x^{n}} \int_{x}^{b}(y-x)^{n-\gamma-1} f(y) d y \quad n-1 \leq \gamma<n,
$$

where $n$ is the smallest integer larger than the number $\gamma$. Moreover, in an infinite domain, $a=-\infty$ or $b=\infty$, the Fourier transforms of the left and right operators, Eq. (19) and Eq. (20), are given by

$$
\mathcal{F}\left\{{ }_{-\infty} D_{x}^{\gamma} f(x)\right\}=(-i \xi)^{\gamma} \hat{f}(\xi) \quad \text { and } \quad \mathcal{F}\left\{{ }_{x} D_{\infty}^{\gamma} f(x)\right\}=(i \xi)^{\gamma} \hat{f}(\xi)
$$

Fractional derivatives are closely related to non-Gaussian stochastic processes. As discussed in Ref. [24] and references therein, these operators naturally appear in the description of anomalous transport in continuous-time (non-Brownian) random walks. In particular, the probability distribution function of random walkers with algebraic decaying jump size $l$, with distribution functions of the form $p \sim l^{-(1+\alpha)}$, is described by fractional diffusion equations of order $\alpha$.

\subsection{Derivation of FMLS FPDE}

According to Eq. (12), the characteristic exponent of the FMLS process, i.e. an LS process with $\beta=-1$, is

$$
\Psi_{F M L S}(-\xi)=-\frac{1}{2} \sigma^{\alpha} \sec (\alpha \pi / 2)(-i \xi)^{\alpha}
$$

and the convexity adjustment of the random walk in Eq. (8) is

$$
v=-\frac{1}{2} \sigma^{\alpha} \sec (\alpha \pi / 2)
$$


Substituting Eq. (22) in Eq. (17) and taking the inverse Fourier transform delivers the pricing FPDE

$\frac{\partial V(x, t)}{\partial t}+\left(r+\frac{1}{2} \sigma^{\alpha} \sec (\alpha \pi / 2)\right) \frac{\partial V(x, t)}{\partial x}-\frac{1}{2} \sigma^{\alpha} \sec (\alpha \pi / 2)_{-\infty} D_{x}^{\alpha} V(x, t)=r V(x, t)$.

\subsection{Derivation of CGMY and KoBoL FPDEs}

If the shocks to the log-stock price are CGMY we obtain the pricing FPDE by substituting $\Psi_{C G M Y}(-\xi)$, using Eq. (14), in Eq. (17). Taking the inverse Fourier transform yields

$$
\begin{gathered}
\frac{\partial V(x, t)}{\partial t}+(r-v) \frac{\partial V(x, t)}{\partial x} \\
+C \Gamma(-Y) e^{M x}{ }_{x} D_{\infty}^{Y}\left(e^{-M x} V(x, t)\right) \\
+C \Gamma(-Y) e^{-G x}{ }_{-\infty} D_{x}^{Y}\left(e^{G x} V(x, t)\right)=\left(r+C \Gamma(-Y)\left(M^{Y}+G^{Y}\right)\right) V(x, t),
\end{gathered}
$$

where

$$
v=C \Gamma(Y)\left\{(M-1)^{Y}-M^{Y}+(G+1)^{Y}-G^{Y}\right\} .
$$

Note that for $Y<0$ the fractional operators in Eq. (25) are fractional integrals.

Similarly, if the risk-neutral log-stock price dynamics follow a KoBoL process, the pricing equation satisfied by European-style options satisfies

$$
\begin{gathered}
\frac{\partial V(x, t)}{\partial t}+\left(r-v-\lambda^{\alpha-1}(q-p)\right) \frac{\partial V(x, t)}{\partial x}+ \\
\frac{1}{2} \sigma^{\alpha}\left[p e^{\lambda x}{ }_{x}^{\alpha} e^{-\lambda x} V(x, t)+q e^{-\lambda x}{ }_{-\infty} D_{x}^{\alpha} e^{\lambda x} V(x, t)\right]=\left(r+\frac{1}{2} \sigma^{\alpha} \lambda^{\alpha}\right) V(x, t),
\end{gathered}
$$

where

$$
v=\frac{1}{2} \sigma^{\alpha}\left\{p(\lambda-1)^{\alpha}+q(\lambda+1)^{\alpha}-\lambda^{\alpha}-\alpha \lambda^{\alpha-1}(q-p)\right\} .
$$

As expected, this equation coincides with the PIDE derived in Ref. [3] for KoBoL processes, and if $\alpha=2$ we recover the classical BS partial differential Eq. (3). 
CGMY and KoBoL processes are particularly useful damped Lévy processes because, as shown here, they lead to generalized fractional operators involving exponential damping factors that can be incorporated into pricing equations. Other possible dampening include power-law cutoffs that can also be incorporated into generalized fractional operators, see Ref. [33]. However, power-law truncations are not suited for derivative pricing because the expectation value of the stock price, $\mathbb{E}^{Q}\left[S_{t}\right]=\mathbb{E}^{Q}\left[e^{X_{t}}\right]$, diverges when the distribution of the random variable $X_{t}$ exhibits algebraic tails for $x>0$. Note that this is not an issue for FMLS processes which involve maximally skewed distributions where only the left tail exhibits algebraic decay and the expectation $\mathbb{E}^{Q}\left[e^{X_{t}}\right]<\infty$, see Ref. [30].

In this section we have shown that for the Lévy processes discussed here, the pricing equations satisfied by European-style derivatives are FPDEs. When it was assumed that log-stock prices follow an FMLS process, we observed, as a consequence of a heavy left tail of the distribution, that only the left RL operator appears. For the CGMY and KoBoL we see that both the right and left RL operators appear as a consequence of both left and right heavy tails of the distribution of log-stock prices.

\section{Option pricing: numerical solution of FPDEs}

In the previous section it was shown that for the Lévy processes discussed in Section 2 , the pricing equation for European-style options can be written using fractional derivative operators. Beyond the conceptual insight gained by doing this, one of the main advantages of introducing explicitly fractional operators rests on the possibility of using recently developed numerical methods for solving fractional order equations. In the standard BS model, the local nature of the differential operators involved, makes the solution of the pricing equation straightforward by using well-understood methods, e.g. finite-difference techniques Ref. [34]. On the other hand, as discussed before, non-Gaussian models lead to pricing equations involving integro-differential operators whose non-local behavior creates non-trivial numerical problems. See for example Ref. [11] and references therein. 
In the case of infinite domains, where boundary conditions are easily incorporated, Fourier transform methods provide an efficient technique for solving integrodifferential pricing equations Ref. [7]. However, transform methods cannot be applied to problems in finite or semi-infinite domains where the operators have to be localized and boundary conditions imposed. An important example of this type of problems are barrier options. In their simplest realization, barrier options, also known as knock-out options, are similar to European calls and puts with the difference that their value depends on the stock price 'hitting' a known threshold throughout the life of the option. The presence of such barrier makes the solution of the option pricing problem dependent on the trajectory of the stock value.

In this section we consider the pricing of knock-out barrier options for FMLS processes using Eq. (24). Depending on the boundary conditions, there are three different cases: up-and-out, down-and-out, and double-knock-out options. For a European up-and-out call option with barrier located at $x=B_{u}$ the boundary conditions are

$$
V(x, t)=\left\{\begin{array}{llr}
0 & \text { for } e^{x} \geq e^{B_{u}}, \quad 0 \leq t<T, \\
\max \left(e^{x}-K, 0\right) & \text { for } 0<e^{x}<e^{B_{u}}, \quad t=T .
\end{array}\right.
$$

Boundary conditions for European down-and-out call options follow a similar logic requiring the value of the option to vanish when the price of the asset falls below a prescribed value, $x=B_{d}$,

$$
V(x, t)=\left\{\begin{array}{lll}
0 & \text { for } e^{x} \leq e^{B_{d}}, & 0 \leq t<T, \\
\max \left(e^{x}-K, 0\right) & \text { for } e^{B_{d}}<e^{x}, & t=T .
\end{array}\right.
$$

Finally, double-knock-out options with lower boundary at $x=B_{d}$ and upper boundary at $x=B_{u}$ require the vanishing of the price when $e^{x} \leq e^{B_{d}}$ and $e^{x} \geq e^{B_{u}}$ for $0 \leq t<T$, with $V(x, T)=\max \left(e^{x}-K, 0\right)$ for $e^{B_{d}}<e^{x}<e^{B_{u}}$. Note that the main difference between jump models and the BS case is that in the former the boundary condition at the barrier must also specify the value of the option beyond the barrier, which is zero. This is the correct specification because the jump nature of the stock price can take the underlying over the barrier without hitting it as in the diffusion case where the underlying stock price has continuous paths. 
The standard Black-Scholes model and its fractional extensions assume an infinite domain, $x \in(-\infty, \infty)$. However, to solve the corresponding equations numerically it is necessary to truncate the original unbounded domain into a finite interval. Whereas this truncation is more or less direct in the standard Black-Scholes case, it is nontrivial in the fractional case due to the non-locality of the operators involved. In this section we apply the truncation method and numerical scheme originally proposed in Refs. $[13,12]$ to solve the fractional Black-Scholes equation for FMLS processes.

A straightforward way to truncate the fractional operators is to approximate ${ }_{-\infty} D_{x}^{\gamma} \approx{ }_{a} D_{x}^{\gamma}$, where $a$ is the lower bound of the finite size domain of interest. In the case of down-and-out options, $a=B_{d}$. However, this naive prescription is problematic because for finite $a$ the left Riemann-Liouville derivative is singular at the lower, $x=a$, boundary. To understand the nature of this singularity consider a differentiable function $f$. Expanding in Taylor series around $x=a$ and fractionaldifferentiating term by term (using Eq. (51)) we get for $1<\gamma<2$,

$$
{ }_{a} D_{x}^{\gamma} f=\frac{1}{\Gamma(1-\gamma)} \frac{f(a)}{(x-a)^{\gamma}}+\frac{1}{\Gamma(2-\gamma)} \frac{f^{\prime}(a)}{(x-a)^{\gamma-1}}+\sum_{k=0}^{\infty} \frac{f^{(k+2)}(a)(x-a)^{k+2-\gamma}}{\Gamma(k+3-\gamma)}
$$

The key observation is that, for $1<\gamma<2$ and finite $a$, the first two terms on the right hand side are in general singular. Rewriting Eq. (31) as

$$
{ }_{a} D_{x}^{\gamma}\left[f(x)-f(a)-f^{\prime}(a)(x-a)\right]=\sum_{k=0}^{\infty} \frac{f^{(k+2)}(a)(x-a)^{k+2-\gamma}}{\Gamma(k+3-\gamma)},
$$

with the regular terms on the right hand side, it is observed that, although the truncated Riemann-Liouville derivative of a general function is singular, the derivative of the function with the boundary terms subtracted is regular. This motivates the definition of the regularized, truncated left fractional derivative of order $1<\gamma<2$ as

$$
{ }_{a}^{c} D_{x}^{\gamma} f={ }_{a} D_{x}^{\gamma}\left[f(x)-f(a)-f^{\prime}(a)(x-a)\right],
$$

which after integration by parts can be written as

$$
{ }_{a}^{c} D_{x}^{\gamma} f=\frac{1}{\Gamma(2-\gamma)} \int_{a}^{x} \frac{\partial_{y}^{2} f}{(x-y)^{\gamma-1}} d y .
$$

We use the left super-index "c" because this regularized fractional derivative corresponds to the Caputo fractional derivative used in the study of fractional derivative 
operators in time, see for example Refs. [27, 29]. By construction, for well-behaved functions, in the limit $x \rightarrow a,{ }_{a}^{c} D_{x}^{\gamma} f \rightarrow 0$, and as expected, in the limit $a \rightarrow-\infty$, ${ }_{a}^{c} D_{x}^{\gamma} f \rightarrow_{-\infty} D_{x}^{\gamma} f$. Based on this, following Refs. [13, 12], for the numerical solution of the fractional BS equation in the $(a, b)$ domain, we truncate the fractional derivative using the approximation ${ }_{-\infty} D_{x}^{\gamma} \approx{ }_{a}^{c} D_{x}^{\gamma}$.

In the numerical solution of the standard and the fractional Black-Scholes models one has to translate the asymptotic boundary conditions into the finite domain of interest. Since the fractional FMLS model contains only a left fractional derivative, non-locality plays no role in the specification of the boundary conditions at $x=B_{u}$ which can be implemented numerically following what is done in the standard BlackScholes model (see for example Ref. [34] ). The boundary conditions at the lower boundary are less trivial to implement since in this case the non-local effects of the left derivative play a role. For down-and-out and double-knock-out options the truncation guarantees by definition the correct boundary condition $V(x, t)=0$ for $x \leq B_{d}$. For up-and-out barriers, we adopt the Neumann boundary condition $V^{\prime}\left(B_{d}, t\right)=0$. This boundary condition neglects the contribution of the fractional derivative from the $\left(-\infty, B_{d}\right)$ interval. However this approximation is justified by the fact that the Greens's function of the fractional Black-Scholes derivatives for an FMLS process is an extremal LS distribution that decays exponentially in the $\left(-\infty, B_{d}\right)$ interval.

A key issue in the solution of PIDEs is the discretization of the integral operator(s) involved. For the case of fractional operators two methods can be followed. One consists of a direct finite different approximation of the integral appearing in the definition of the RL derivative. This method, which can in principle be applied to general PIDEs, was used in Ref. [17] to solve FPDEs. An alternative method is based on the Grünwald-Letnikov (GL) definition of the fractional derivative according to which

$$
{ }_{a} D_{x}^{\gamma} f(x)=\lim _{h \rightarrow 0} \frac{-\Delta_{h}^{\gamma} f(x)}{h^{\gamma}},
$$

where the left finite-difference fractional operator, ${ }^{-} \Delta_{h}^{\mu} f$, is defined as

$$
{ }^{-} \Delta_{h}^{\gamma} f(x)=\sum_{j=0}^{m_{-}} w_{j}^{(\gamma)} f(x-j h),
$$

with $m_{-}=[(x-a) / h]$, where the brackets [ ] denote the integral part, Ref. [27]. 
The right fractional derivative is defined in an analogous manner. In the first order approximation, the coefficients $w_{j}^{(\mu)}$ are recursively defined as

$$
w_{0}^{(\gamma)}=1, \quad w_{k}^{(\gamma)}=\left(1-\frac{\gamma+1}{k}\right) w_{k-1}^{(\gamma)}, \quad k=1,2, \ldots N,
$$

and for finite $h$ they provide a first order approximation of the fractional derivative, i.e.

$$
{ }_{a} D_{x}^{\gamma} V-\frac{{ }_{a} \Delta_{h}^{\gamma} V}{h^{\gamma}}=O(h) .
$$

For well-behaved functions it can be shown that the Grünwald-Letnikov definition is equivalent to the Riemann-Liouville definition, see for example Ref. [27].

For the numerical integration we use the "backward" time variable $T-t$ so that the payoff curve at expiry gives the initial condition at $T-t=0$ and the evolution of the price follows a diffusion-like process for $T-t>0$. The integration domain $x \in\left(B_{d}, B_{u}\right)$ is divided into $N$ equally spaced segments with grid points at $\left\{x_{k}\right\}$ for $k=0,1, \ldots N$, with $x_{0}=B_{d}, x_{N}=B_{u}$, and $x_{k+1}-x_{k}=\left(B_{u}-B_{d}\right) / N=h$. The value of $V$ at grid point $x_{k}$ is denoted as $V_{k}$. The first order regular derivative in the FPDE is discretized using an up-wind scheme Ref. [26]. Following Refs. [13, 12], to discretize the left fractional derivative, we first write the operator in flux conserving form ${ }_{a} D_{x}^{\gamma}=\partial_{x a} D_{x}^{\gamma-1}$. The first order derivative $\partial_{x}$ is then discretized using a forward finite-difference scheme and the fractional derivative of order $\gamma-1$ is discretized using the Grünwald-Letnikov representation. The resulting finite-difference equation can be written in matrix form as

$$
\partial_{t} V_{k}=h^{-\gamma}[M V]_{k} .
$$

For the time advance we use the weighted average method

$$
V_{k}^{m+1}-V_{k}^{m}=\nu \Lambda[M V]_{k}^{m+1}+\nu(1-\Lambda)[M V]_{k}^{m}
$$

where $V_{k}^{m}$ denotes the value of $V$ at grid point $k$ at time $t=m \Delta t$, and $\nu=\Delta t / h^{\gamma}$. Solving for $V^{m+1}$ leads to

$$
V_{k}^{m+1}=[1-\nu \Lambda M]^{-1}[1-\nu(1-\Lambda) M] V_{k}^{m} .
$$

The weighting factor $\Lambda$ can in general depend on $\gamma$ but for the calculation presented here we used the Crank-Nicolson prescription $\Lambda=1 / 2$. Further details of the numerical method can be found in Refs. [13, 12]. 
In the calculations reported here to price barrier options, it is assumed that the options are struck at $K=50$ and the starting value of the stock price at time $t=0$ is $S_{0}=50$. The down barrier is located at $B_{d}=30$, and the up barrier is located at $B_{u}=83$. Figure 1 shows the up-and-out values; Figure 2 the down-and-out values; and Figure 3 the double-knock-out values for the FMLS FDE with $\alpha=1.5$ and $\sigma=0.25$. All figures show values for $T=\{3 / 12,2 / 12,1 / 12,2 / 52,1 / 52,0\}$. That is, 3 months, 2 months, 1 month, 2 weeks, 1 week and at expiry. To interpret these results we evaluate the difference between the prices obtained by a trader who assumes a BS model and a trader who assumes that the log-stock process follows an FMLS process. For illustrative purposes we assume that the two traders' measure of the variance of returns, $\left(S_{t+\Delta t}-S_{t}\right) / S_{t}$, over a time step $\Delta t$ coincide. According to this prescription $\sigma=0.25$ in the FMLS implies $\sigma_{B S}=0.2706$ in the BS model. Figures 4, 5 and 6 show the corresponding difference between the BS and FMLS values. It is interesting to observe that for the three types of knock-out call options considered, the BS model delivers higher prices when $S<K$ and lower prices for deeper in-themoney options $(S \gtrsim 53)$. In the up-and-out case it is straightforward to see that the jump nature of the FMLS process must deliver a much higher price for in-themoney options than the equivalent BS case. As mentioned above, the FMLS process exhibits downwards jumps but no upwards jumps. Therefore, compared to Gaussian shocks, the probability of hitting the up barrier is much lower than if shocks were Gaussian. Finally, when the barrier is placed below the strike, BS options are more expensive than FMLS for out-of-the-money down-and-out options, and cheaper for in-the-money options. Note that in this case, the presence of downward jumps in the FMLS reduces the value of an FMLS down-and-out call but it is still more expensive (for in-the-money values) than the BS down-and-out. Finally, Figure 6 shows the difference in prices for the double knock-out that may be interpreted in the same way the up-and-out and down-and-out discussed above. 


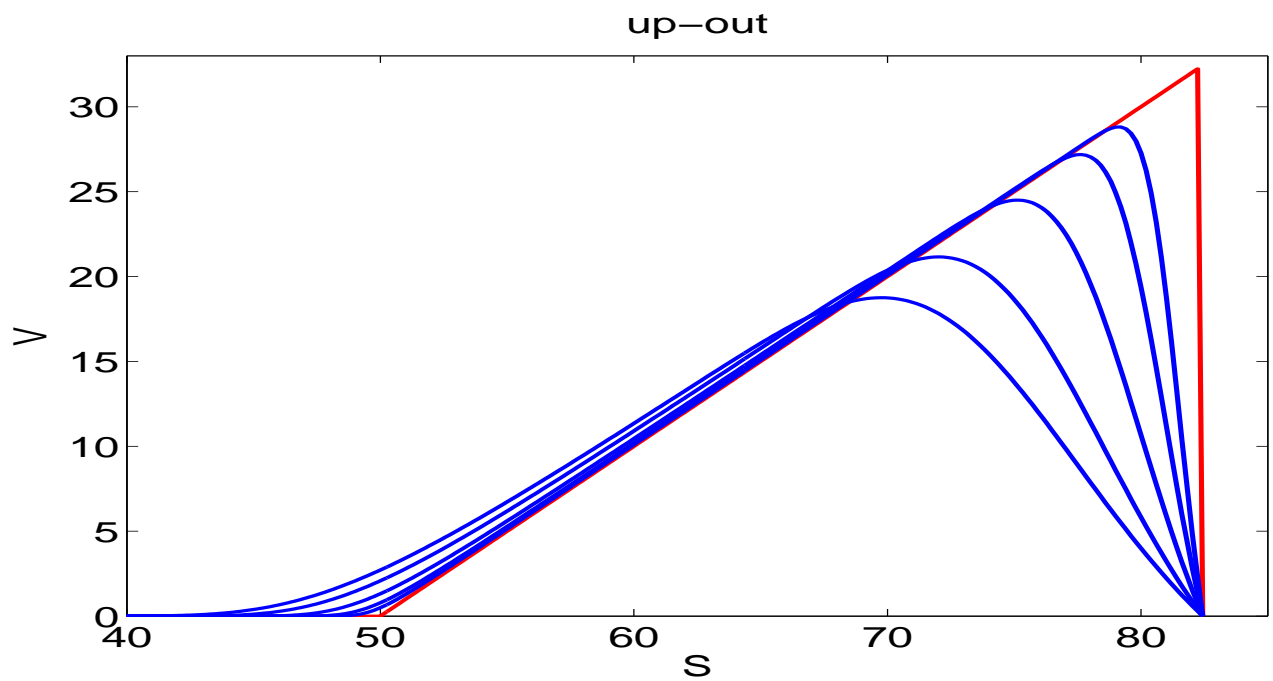

Figure 1: FMLS Up-and-Out. Up-and-Out values with $\alpha=1.5, B_{u}=83$ and $\sigma=0.25$ for $T=\{3 / 12,2 / 12,1 / 12,2 / 52,1 / 52,0\}$.

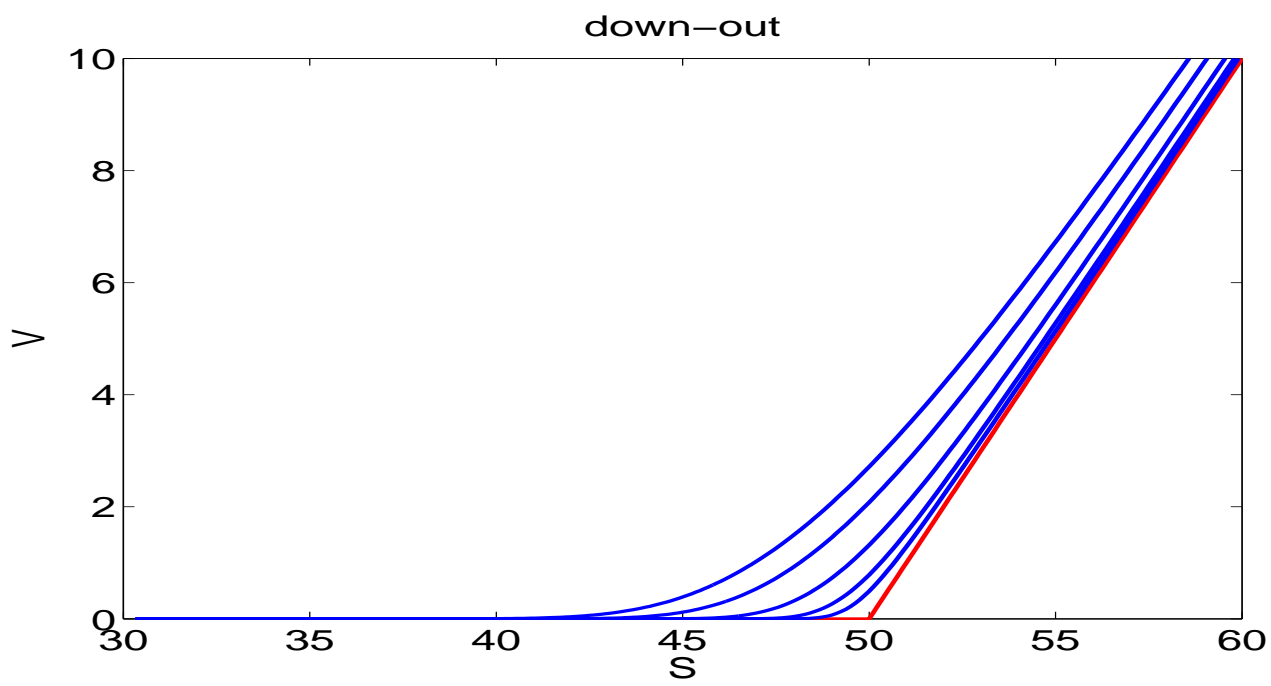

Figure 2: FMLS Down-and-Out. Down-and-Out values with $\alpha=1.5, B_{d}=43$ and $\sigma=0.25$ for $T=\{3 / 12,2 / 12,1 / 12,2 / 52,1 / 52,0\}$.

\section{Conclusions and further work}

Over the past decade, the financial literature has proposed a multitude of different models to capture the dynamics of financial assets. The use of Lévy processes has 


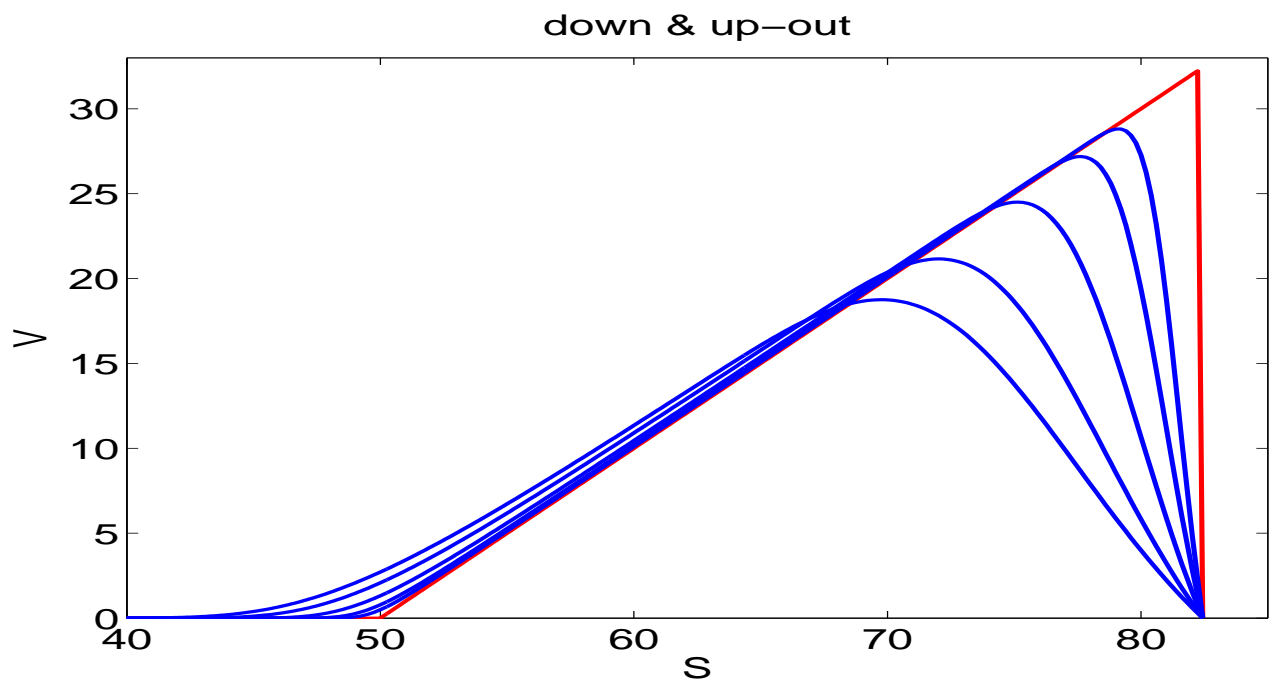

Figure 3: FMLS Double-Knock-Out. Double-Knock-Out values with $\alpha=1.5$, $B_{u}=83, B_{d}=30, \sigma=0.25$ and for $T=\{3 / 12,2 / 12,1 / 12,2 / 52,1 / 52,0\}$.

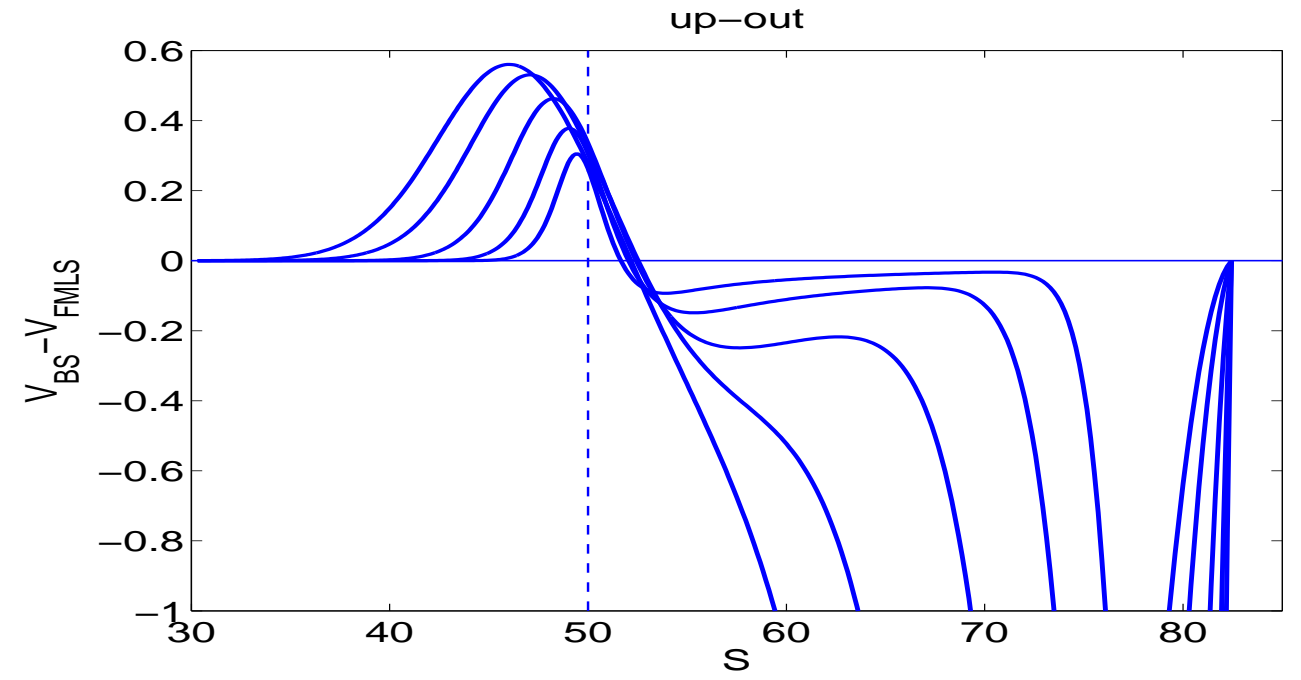

Figure 4: Black-Scholes vs FMLS Up-and-Out. Difference in Up-and-Out values with $\alpha=1.5, B_{u}=83, \sigma=0.25$ and $\sigma_{B S}=0.2706$ for $T=$ $\{3 / 12,2 / 12,1 / 12,2 / 52,1 / 52,0\}$.

proven to be an excellent tool that strikes the right balance between capturing the desired properties of stock price evolution and mathematical tractability. The class of Lévy processes is vast, but for equity modeling, the FMLS, CGMY and KoBoL stand 


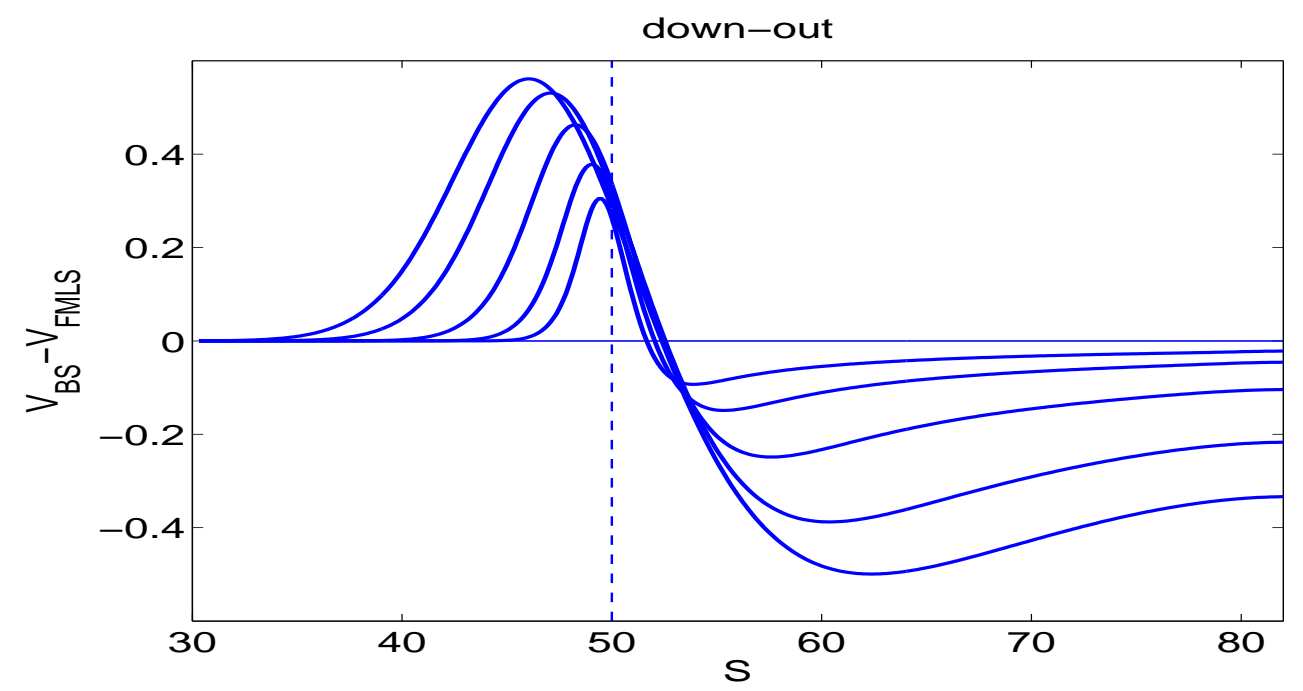

Figure 5: Black-Scholes vs FMLS Down-and-Out. Difference in Down-andOut values with $\alpha=1.5, B_{d}=43, \sigma=0.25$, and $\sigma_{B S}=0.2706$ for $T=$ $\{3 / 12,2 / 12,1 / 12,2 / 52,1 / 52,0\}$.

out as some of the best choices among practitioners and academics. On the other hand the use of fractional operator theory seems to be increasing in a number of disciplines. In this article we show that financial instruments that derive their value from assets that are modeled as geometric Lévy processes, such as those mentioned above, satisfy FPDEs. These fractional equations may be used not only to price simple options, such as European calls and puts, but can also be employed to solve other more exotic instruments, such as barrier options, and American options. To illustrate this we priced barrier options when the stock price follows a geometric FMLS process.

The pricing of American options may also be performed numerically by noting that instead of having equality we have an inequality in the FPDEs. For example, an American option written on an asset that follows a geometric KoBoL process, satisfies

$$
\begin{gathered}
\frac{\partial V(x, t)}{\partial t}+\left(r-v-\lambda^{\alpha-1}(q-p)\right) \frac{\partial V(x, t)}{\partial x} \\
+\frac{1}{2} \sigma^{\alpha}\left[p e^{\lambda x}{ }_{x}^{\alpha} e^{-\lambda x} V(x, t)+q e^{-\lambda x}{ }_{-\infty} D_{x}^{\alpha} e^{\lambda x} V(x, t)\right] \leq\left(r+\frac{1}{2} \sigma^{\alpha} \lambda^{\alpha}\right) V(x, t),
\end{gathered}
$$

where $v$ is given by Eq. (28) and subject to the relevant boundary conditions. The 


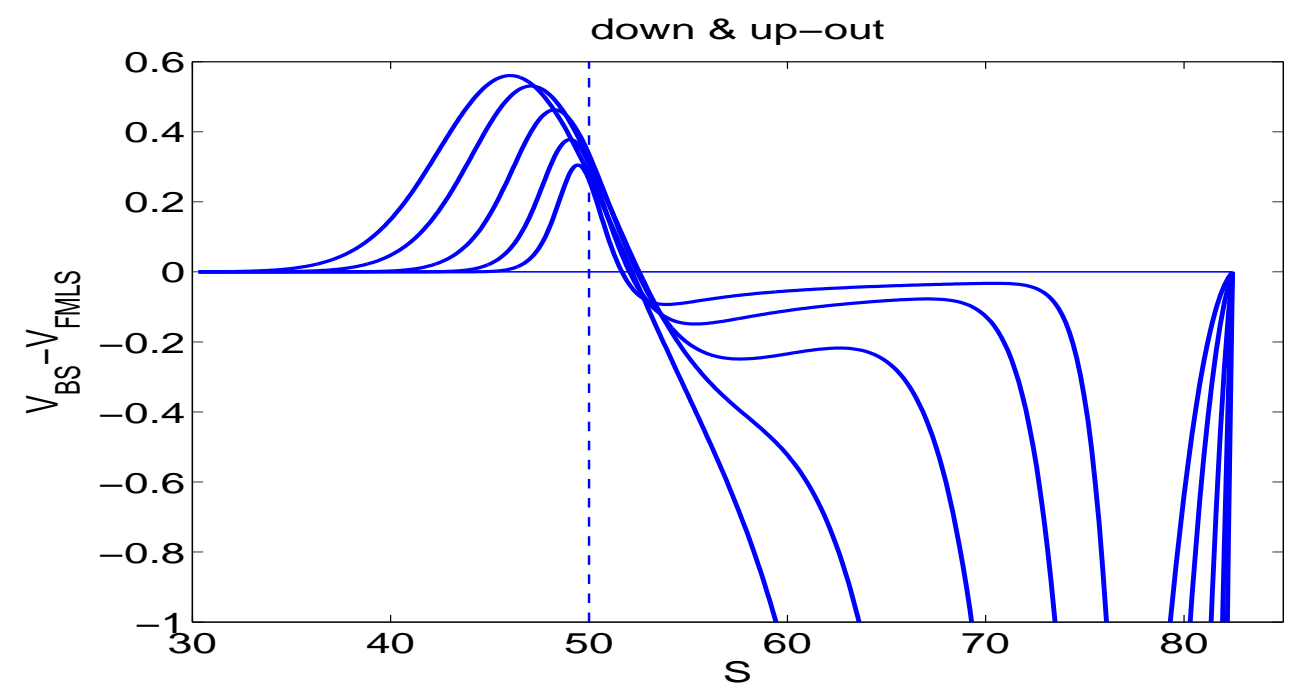

Figure 6: Black-Scholes vs FMLS Double Knock-Out. Difference Double Knock-Out values with $\alpha=1.5, B_{u}=83, B_{d}=30, \sigma=0.25$ and $\sigma_{B S}=0.2706$ for $T=\{3 / 12,2 / 12,1 / 12,2 / 52,1 / 52,0\}$.

case $T=\infty$ is known as a perpetual option and closed-form solutions may be obtained for a for a large class of members of the Lévy processes family Refs. [4, 25].

Finally, as for American options, there is a need to be able to price more complex instruments and in the majority of these cases one has to resort to numerical techniques that involve solving pricing equations, such as the ones derived here, subject to the relevant boundary and initial conditions. It is here that the wealth of literature and techniques developed in the field of fractional operators can be applied to price a wide range of financial instruments.

\section{Acknowledgment}

The authors are grateful to Pablo Padilla for useful discussions on the applications of fractional calculus in finance. Cartea acknowledges financial support from the Nuffield Foundation NAL/00791/G. 


\section{A Derivation of Eq. (17)}

Here we show that the Fourier transform of the value of a European-style option written on an asset that follows Eq. (8) satisfies Eq. (17). We start by writing the value of the option as the risk-neutral expectation of the final payoff $\Pi\left(x_{T}, T\right)$

$$
V(x, t)=e^{-r(T-t)} \mathbb{E}^{Q}\left[\Pi\left(x_{T}, T\right)\right] .
$$

Assuming that the payoff $\Pi\left(x_{T}, T\right)$ has a complex Fourier transform

$$
\mathcal{F}\left\{\Pi\left(x_{T}, T\right)\right\} \equiv \hat{\Pi}(\xi, T)=\int_{-\infty+i \xi_{i}}^{\infty+i \xi_{i}} e^{i \xi x_{T}} \Pi\left(x_{T}, T\right) d x_{T},
$$

in the strip $a<\xi_{i}<b$, where we denote $\xi_{i}=\operatorname{Im} \xi$, we write

$$
V(x, t)=\frac{e^{-r(T-t)}}{2 \pi} \mathbb{E}^{Q}\left[\int_{-\infty+i \xi_{i}}^{\infty+i \xi_{i}} e^{-i x_{T} \xi} \hat{\Pi}(\xi, T) d \xi\right] .
$$

Taking the expectation operator inside the integral, see Ref. [16], we obtain

$$
\begin{aligned}
V(x, t) & =\frac{e^{-r(T-t)}}{2 \pi} \int_{-\infty+i \xi_{i}}^{\infty+i \xi_{i}} \mathbb{E}^{Q}\left[e^{-i x_{T} \xi}\right] \hat{\Pi}(\xi, T) d \xi \\
& =\frac{e^{-r(T-t)}}{2 \pi} \int_{-\infty+i \xi_{i}}^{\infty+i \xi_{i}} e^{-i \xi x_{t}-i \xi(r-v)(T-t)} e^{(T-t) \Psi(-\xi)} \hat{\Pi}(\xi, T) d \xi
\end{aligned}
$$

where $\Psi(\xi)$ is the characteristic exponent of the Lévy process $L_{t}$. Note that we require $e^{\Psi(-\xi)}$ to be analytic in a strip that intersects the strip where the complex Fourier transform of the payoff exists. It follows from Eq. (44) that

$$
\hat{V}(\xi, t)=e^{[-r-i \xi(r-v)+\Psi(-\xi)](T-t)} \hat{\Pi}(\xi, T),
$$

which is the solution of Eq. (17) with boundary condition $\hat{V}(\xi, T)=\hat{\Pi}(\xi, T)$.

\section{B Review of fractional calculus}

In this appendix we review some useful results from fractional calculus pertaining to the present paper. Further information can be found in Refs. [27, 29]. A convenient 
way to define the fractional derivative is by first introducing the fractional integral. Let $f(x)$ be a real valued function, and $n$ an integer number. Then, the $n$-th order integration of $f(x)$ is

$$
{ }_{a} D_{x}^{-n} f(x)=\int_{a}^{x} d x_{1} \int_{a}^{x_{1}} d x_{2} \ldots \int_{a}^{x_{n-1}} d x_{n} f\left(x_{n}\right),
$$

where $a$ is a constant. Equation (45) can equivalently be written as

$$
{ }_{a} D_{x}^{-n} f(x)=\frac{1}{(n-1) !} \int_{a}^{x} \frac{f(y)}{(x-y)^{1-n}} d y \text {. }
$$

A straightforward extension of Eq. (45) to non-integer order $\gamma$ leads to

$$
{ }_{a} D_{x}^{-\gamma} f(x)=\frac{1}{\Gamma(\gamma)} \int_{a}^{x} \frac{f(y)}{(x-y)^{1-\gamma}} d y,
$$

where $\Gamma$ is the gamma function, which generalizes the factorial to non-integer values. Equation (47) is the Riemann-Liouville fractional integral of order $\gamma$. Results from regular integral calculus extend naturally to this operator. An instructive example is the fractional integral of a power

$$
{ }_{0} D_{x}^{-\gamma} x^{\mu}=\frac{\Gamma(\mu+1)}{\Gamma(\mu+\gamma+1)} x^{\mu+\gamma}
$$

Based on the fractional integral, the fractional derivative of order $\gamma$ is defined as

$$
{ }_{a} D_{x}^{\gamma} f(x)=\frac{\partial^{m}}{\partial x^{m}}\left[{ }_{a} D_{x}^{-(m-\gamma)} f(x)\right],
$$

where $m$ is the smallest integer greater than $\gamma$. As expected, for integer $\gamma=N$, ${ }_{a} D_{x}^{N} f(x)=\partial^{N} f(x) / \partial x^{N}$. Substituting Eq. (47) into Eq. (49) leads to RiemannLiouville fractional derivative of order $\gamma$,

$$
{ }_{a} D_{x}^{\gamma} f(x)=\frac{1}{\Gamma(m-\gamma)} \frac{\partial^{m}}{\partial x^{m}} \int_{a}^{x} \frac{f(y)}{(x-y)^{\gamma+1-m}} d y,
$$

where $m-1 \leq \gamma<m$ with $m$ a positive integer. As an example,

$$
{ }_{0} D_{x}^{\gamma} x^{\mu}=\frac{\Gamma(\mu+1)}{\Gamma(\mu-\gamma+1)} x^{\mu-\gamma} .
$$

As in the case of the fractional integral, basic results from regular calculus naturally extend to the fractional derivative operator. 
The value of the fractional derivative in Eq. (50) at $x$ depends on the behavior of the function $f(x)$ to the "left" of $x$, i.e. in the interval $(a, x)$. This is the reason why, in a more precise terminology, Eq. (50) is called the left Riemann-Liouville fractional derivative. The right Riemann-Liouville fractional derivative is naturally defined by switching the integration limits

$$
{ }_{x} D_{b}^{\gamma} f(x)=\frac{1}{\Gamma(m-\gamma)} \frac{\partial^{m}}{\partial x^{m}} \int_{x}^{b} \frac{f(y)}{(y-x)^{\gamma+1-m}} d y .
$$

The general fractional derivative operator is defined as a superposition of the left and right derivatives. Moreover, in an infinite domain, $a=-\infty$ or $b=\infty$, the Fourier transforms of the left and right operators are given by

$$
\mathcal{F}\left\{{ }_{-\infty} D_{x}^{\gamma} f(x)\right\}=(-i \xi)^{\gamma} \hat{f}(\xi) \quad \text { and } \quad \mathcal{F}\left\{{ }_{x} D_{\infty}^{\gamma} f(x)\right\}=(i \xi)^{\gamma} \hat{f}(\xi)
$$




\section{References}

[1] T. Björk. Arbitrage Theory in Continuous Time. Oxford University Press, 1st edition, 1998.

[2] S. Boyarchenko and S. Levendorskii. Barrier options and touch-and-out options under regular Lévy processes of exponential type. Annals of Applied Probability, 12(4):1261-1298, 2002.

[3] S. Boyarchenko and S. Levendorskii. Non-Gaussian Merton-Black-Scholes Theory, volume 9. World Scientific, 2002.

[4] S. Boyarchenko and S. Levendorskii. Perpetual American options under Lévy processes. SIAM Journal on Control and Optimization, 40:1663-1696, 2002.

[5] S. Boyarchenko and S. Levendorskii. Generalization of the Black-Scholes equation for truncated Lévy processes. Working paper, 2006.

[6] P. Carr, H. Geman, D. Madan, and M. Yor. The fine structure of asset returns: an empirical investigation. Journal of Business, 75(2):305-332, 2002.

[7] P. Carr and D. Madan. Option valuation using the fast Fourier transform. Journal of Computational Finance, 2:61-73, 1999.

[8] P. Carr and L. Wu. The finite moment logstable process and option pricing. The Journal of Finance, LVIII(2):753-777, April 2003.

[9] Á. Cartea. Dynamic hedging of financial instruments when the underlying follows a non-Gaussian process. Working Paper, Birkbeck College, University of London, 2005.

[10] R. Cont and P. Tankov. Financial Modelling With Jump Processes. Chapman and Hall, 2004.

[11] R. Cont and E. Voltchkova. Finite difference methods for option pricing in jumpdiffusion and exponential Lévy models. SIAM Journal of Numerical Analysis, 43(4):1596-1626, 2005. 
[12] D. del-Castillo Negrete. Finite difference methods for fractional diffusion equations. Working paper, 2006.

[13] D. del-Castillo Negrete. Fractional diffusion models of non-local transport. Accepted for publication in Phys. of Plasmas, 2006.

[14] W. Feller. An Introduction to Probability Theory and its Applications, volume II. Wiley, 1966.

[15] I. Koponen. Analytic approach to the problem of convergence of truncated Levy flights towards the Gaussian stochastic process. Physical Review E, 52:11971199, November 1995.

[16] A. Lewis. A simple option formula for general jump-diffusion and other exponential Lévy processes. Working Paper, September 2001.

[17] V. Lynch, B. Carreras, and D. del-Castillo-Negrete. Numerical methods for the solution of partial differential equations of fractional order. J. Comp. Phys., pages 406-421, 2003.

[18] F. Mainardi, M. Raberto, R. Gorenflo, and E. Scalas. Fractional calculus and continuous-time finance II: the waiting-time distribution. Physica A, 287:469481, December 2000.

[19] B. Mandelbrot. Fractals and Scaling in Finance. Springer, 1st edition, 1997.

[20] R.M. Mantegna and H.E. Stanley. Stochastic processes with ultraslow convergence to a Gaussian: The truncated Levy flight. Physical Review Letters, 73:2946-2949, November 1994.

[21] R.N. Mantegna and H.E. Stanley. Scaling behavior in the dynamics of an economic index. Nature, (376):46-49, 1995.

[22] R.N. Mantegna and H.E Stanley. An Introduction to Econophysics, correlations and complexity in finance. Cambridge, 1st edition, 2000.

[23] R. Merton. Continuous-Time Finance. Blackwell, 1st edition, 1990. 
[24] R. Metzler and J. Klafter. The random walk guide to anomalous diffusion: a fractional dynamics approach. Phys. Reports, 339, 2000.

[25] E. Mordecki. Optimal stopping and perpetual options for Lévy processes. Finance and Stochastics, VI(4):473-493, 2002.

[26] K.W. Morton and D.F. Mayers. Numerical Solutions of Partial Differential Equations. Cambridge University Press, 1996.

[27] I. Podlubny. Fractional Differential Equations, volume 198 of Mathematics in Science and Engineering. Academic Press, San Diego, California, 1st edition, 1999.

[28] S.J. Press. A compound events model for security prices. Journal of Business, 40:317-335, 1967.

[29] S.G. Samko, A.A. Kilbas, and O.I. Marichev. Fractional Integrals and Derivatives, theory and applications. Gordon and Breach Science Publishers, 1993.

[30] G. Samorodnitsky and M. Taqqu. Stable Non-Gaussian Random Processes, Stochastic Models with Infinite Variance. Stochastic Modelling. Chapman Hall, 1st edition, 1994.

[31] E. Scalas, R. Gorenflo, and F. Mainardi. Fractional calculus and continuous-time finance. Physica A, 284:376-384, September 2000.

[32] W. Schoutens. Lévy Processes in Finance. Wiley Series in Probability and Statistics. Wiley, England, 1st edition, 2003.

[33] I.M. Sokolov, A.V. Chechkin, and J. Klafter. Fractional diffusion equation for a power-law-truncated Lévy process. Physica A, 336, 2004.

[34] P. Wilmott, S. Howison, and J. Dewynne. The Mathematics of Financial Derivatives, a student introduction. Cambridge University Press, 1st edition, 1995. 